\title{
Inheritance of Cell-Cycle Duration in the Presence of Periodic Forcing
}

\author{
Noga Mosheiff \\ The Racah Institute of Physics, The Hebrew University of Jerusalem, Jerusalem 91904, Israel \\ Bruno M. C. Martins \\ Sainsbury Laboratory, University of Cambridge, Bateman Street, Cambridge CB2 1LR, United Kingdom \\ Sivan Pearl-Mizrahi \\ The Racah Institute of Physics, The Hebrew University of Jerusalem and Department of Microbiology \\ and Molecular Genetics, IMRIC, The Hebrew University Hadassah Medical School, \\ Jerusalem 91120, Israel \\ Alexander Grünberger \\ Forschungszentrum Jülich, IBG-1: Biotechnology, 52425 Jülich, Germany \\ and Bielefeld University, Multiscale Bioengineering, 33615 Bielefeld, Germany \\ Stefan Helfrich \\ University of Konstanz, Bioimaging Center, 78457 Konstanz, Germany \\ Irina Mihalcescu \\ Université Grenoble Alpes, LIPHY, F-38000 Grenoble, France \\ Dietrich Kohlheyer \\ Forschungszentrum Jülich, IBG-1: Biotechnology, 52425 Jülich, Germany \\ and RWTH Aachen University, Microscale Bioengineering (AVT.MSB), 52074 Aachen, Germany \\ James C. W. Locke \\ Sainsbury Laboratory, University of Cambridge, Bateman Street, Cambridge CB2 1LR, United Kingdom; \\ and Department of Biochemistry, University of Cambridge, \\ Downing Site, Cambridge CB2 1QW, United Kingdom \\ and Microsoft Research, 21 Station Road, Cambridge CB1 2FB, United Kingdom \\ Leon Glass \\ Department of Physiology, 3655 Promenade Sir William Osler, McGill University, \\ Montreal, Quebec, Canada H3G 1 Y6 \\ Nathalie Q. Balaban \\ The Racah Institute of Physics, Hebrew University, Jerusalem 91904, Israel
}

(Received 1 October 2017; revised manuscript received 4 February 2018; published 9 May 2018)

Periodic forcing of nonlinear oscillators leads to a large number of dynamic behaviors. The coupling of the cell cycle to the circadian clock provides a biological realization of such forcing. A previous model of forcing leads to nontrivial relations between correlations along cell lineages. Here, we present a simplified two-dimensional nonlinear map for the periodic forcing of the cell cycle. Using high-throughput single-cell microscopy, we have studied the correlations between cell-cycle duration in discrete lineages of several different organisms, including those with known coupling to a circadian clock and those without known coupling to a circadian clock. The model reproduces the paradoxical correlations and predicts new features that can be compared with the experimental data. By fitting the model to the data, we extract the important parameters that govern the dynamics. Interestingly, the model reproduces bimodal distributions for

**nathalie.balaban@mail.huji.ac.il

Published by the American Physical Society under the terms of the Creative Commons Attribution 4.0 International license. Further distribution of this work must maintain attribution to the author(s) and the published article's title, journal citation, and DOI. 
cell-cycle duration, as well as the gating of cell division by the phase of the clock, without having been explicitly fed into the model. In addition, the model predicts that circadian coupling may increase cell-tocell variability in a clonal population of cells. In agreement with this prediction, deletion of the circadian clock reduces variability. Our results show that simple correlations can identify systems under periodic forcing and that studies of nonlinear coupling of biological oscillators provide insight into basic cellular processes of growth.

DOI: 10.1103/PhysRevX.8.021035

\section{INTRODUCTION}

The process of cell division has fascinated scientists since the invention of the microscope. During the process of cell division in organisms that divide symmetrically, a cell generates two almost identical copies of itself. Many mechanisms act in concert to enhance the fidelity of replication and division, including proofreading, DNA repair enzymes, an elaborate partitioning apparatus, and feedbacks. The statistics of the cell division process in single cells has been proposed to provide an unbiased way to uncover the type of feedback that controls the process of cell division ([1,2] and references therein) and has motivated researchers to gather as much data as possible. Powell, one of the pioneers of single-cells measurements, was described as sitting in a $37^{\circ} \mathrm{C}$ room for many hours watching bacteria divide and recording manually celldivision events [3]. Recent technological advances in microscopy and microfluidics [4,5] have boosted our ability to gather information over tens of thousands of cells and opened the door to quantitative analyses of the process of cell division on lineages [6,7].

Cell division is a discrete process (Fig. 1 and Supplemental Material [8] for Video 1) that occurs at each generation. The cellular components inherited from the previous division govern the state of the cell at birth. Therefore, it is appealing to describe this process with discrete maps. For maps, consecutive timing of key events such as the duration of sleep [9], phase of cardiac firing in a stimulation cycle [10], or cell-cycle duration [11], depend in an iterative manner on the timing of previous events. In most studies involving maps, one assumes that the map is identical under subsequent iterations. For example, for the duration of the cell-cycle $T_{n}$ measured at generation $n$, the iterative map $f$ will be of the form $T_{n}+1=f\left(T_{n}\right)$. Interestingly, assuming cell division can be described by a map, the map itself would be duplicated at each generation. This duplication would not contain new information in the absence of a noise term. However, noise is always present and its result is that, at each generation, an additional branch is generated with slightly different initial conditions, a remarkable feature of self-replication $[11,12]$. Thus, the information contained in the lineage is much larger than on a single branch $[13,14]$. Rich dynamics can emerge from the iterative processes, but these dynamics have rarely been analyzed on lineages [12]. Thus, the study of cell lineages
Subject Areas: Biological Physics, Nonlinear Dynamics

introduces a new class of problems involving dynamics of nonlinear maps that are themselves duplicated with noise in subsequent generations. Our goal is to explore the dynamics of cell-cycle inheritance measured on lineages of single cells (Fig. 1). For this purpose, we focus on the measurement of the duration of the cell cycle, which is the time between two consecutive cell divisions, and its inheritance along lineages that generates the time series $T_{n}$ and its branches. We expand on our previous work analyzing the correlations in cell-cycle duration in mouse lymphocytes [11] and compare high throughput single-cell microscopy data from different organisms, with and without putative periodic forcing. We analyze various measurements such as the correlations between cells in the lineages, the distributions of cell-cycle duration, and the distribution of phase at birth using a discrete two-dimensional map representing the periodic forcing of the cell cycle by an external oscillator.

(a)

(b)
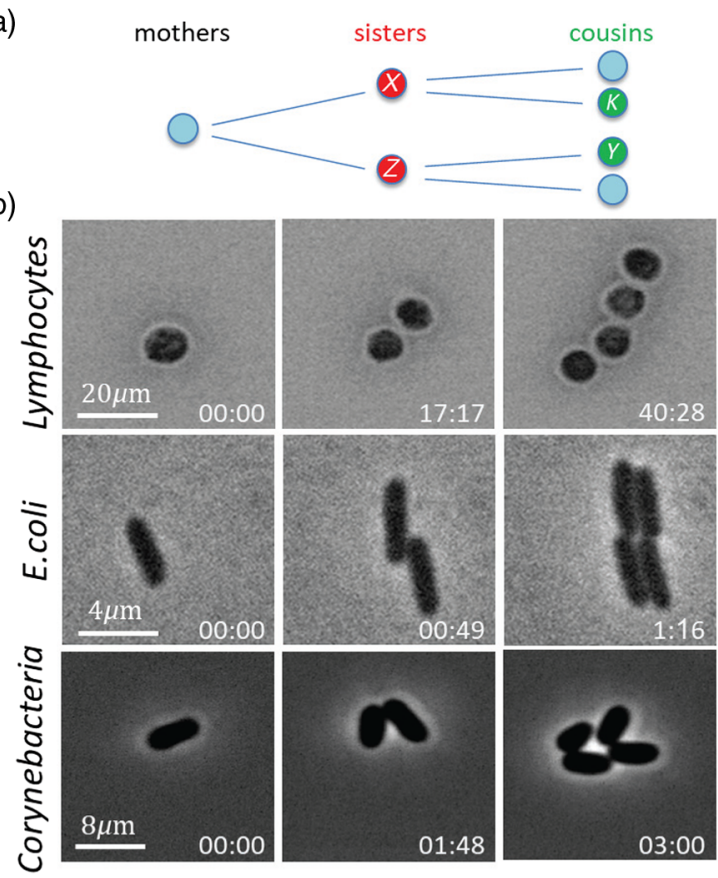

FIG. 1. (a) Schematic illustration of the symmetric selfreplication process that generates two nearly identical sister cells at each division, with sister cells in red (middle) and cousin cells in green (on the right). (b) Single-cell phase-contrast images of the cell-cycle duration in various cells. The time labels are in hours. 


\section{A. Periodic forcing}

One of the classic problems in mathematics involves the effects of period forcing of a nonlinear oscillator [15]. The nonlinear oscillator can be represented by a differential equation containing a stable limit cycle. The periodic forcing is typically either a continuous periodic input or a pulsatile stimulus. In either case, one expects the appearance of certain universal features that can often arise independently of the detailed equations or the nature of the stimuli. If the intrinsic period of the driven oscillator is sufficiently close to the period of the forcing oscillator, then there will typically be entrainment or $1: 1$ phase locking where the two oscillators are synchronized. As one changes the relative frequencies of the oscillators and the strength of influence of the periodic forcing on the driven oscillator, then many different behaviors can arise. One such behavior is $n: m$ phase locking, in which there is a stable periodic rhythm with $n$ cycles of the forcing oscillator to $m$ cycles of the driven oscillator. Two different types of aperiodic rhythms are possible. These can be distinguished by considering what happens to the trajectories starting from two different nearby phases of the driven oscillator as time proceeds. If the distance between the two trajectories grows with time, then the dynamics are chaotic, and if the distance stays approximately the same, then the dynamics are quasiperiodic [16]. Moreover, many features of the locking zones have a universal structure. For low forcing amplitudes, there are typically zones of stable phase locking, called Arnold tongues, where the ratio $m / n$ monotonically increases as the ratio of the forcing oscillator period to the intrinsic period of the forced oscillator increases. These basic insights emerge from research stretching back to Poincaré, with major insights from Arnold [17], Smale [18], and many others. Study of periodically forced nonlinear oscillators is not only of interest in itself, but it can also help us understand dynamics in a wide range of physical [15,19,20] and biological systems [10,21-23].

One such problem involves the effects of an external rhythm on the cell-cycle duration $[11,23,24]$. This external rhythm can be externally imposed, leading to new insight on the cell cycle itself [25], or originate from a clock encoded within the cell, such as the circadian clock [26]. The circadian clock is an internal cellular oscillator that has an approximate period of $24 \mathrm{~h}$ and that can influence cellular processes depending on the time of the day [26]. For example, in some organisms that have a circadian clock, there are time intervals in the day during which the progression through the cell cycle proceeds slowly. This phenomenon is called gating $[27,28]$. It is widely believed that, in the presence of a gate, cell-cycle states synchronize to the circadian signal [23]. Since biological systems typically have considerable variability, even in situations in which there is believed to be synchronization, there can be considerable variation in the timing of the events, and quantitative analysis and modeling are needed to test hypotheses.

Two types of theoretical models have been proposed to interpret the experimental results on entrainment of oscillations: nonlinear differential equations and nonlinear maps. Nonlinear differential equations are often developed specifically for particular systems with parameters generally determined by optimizing fits to data $[23,29]$. In situations in which the differential equations are based on realistic models, as, for example, in models of the interactions of the circadian clock and the cell division cycle [23], it is possible to determine some of the parameters based on different sets of experiments than those used to model entrainment data [24]. Maps constitute an alternative type of model.

In what follows, we discuss the synchronization of the cell cycle to the circadian rhythm in the context of nonlinear dynamics models of periodic forcing. In order to simplify previous approaches and reduce the number of parameters, we apply a stochastic nonlinear map to study cell-cycle time correlations in lineages from several different species. The analysis demonstrates the importance of deterministic nonlinear factors in controlling the cell cycle and also suggests new directions for theoretical analyses. In Sec. II, we present the mathematical model of periodic forcing. Section III gives the experimental results, mathematical analysis of a null model, and the fitting of the mathematical model of periodic forcing to the data. Section IV is the discussion of the results, and in Sec. V we present the experimental methods.

\section{MATHEMATICAL MODEL}

In recent work, some of us proposed a biologically plausible model for the interaction of the circadian rhythm with the cell cycle, called the "kicked cell cycle" [11], which we expand on below. This model is based on the assumption that the cycle duration of a daughter cell depends linearly on the cycle duration of the mother cell, as well as on the circadian time of the cell division. The basic idea is that the cell-cycle duration $T_{n}$ of a cell in generation $n$ can be influenced both by the cell-cycle duration of its mother $T_{n-1}$ and the phase at its birth of a forcing oscillator such as the circadian clock. Given the birth time of a cell in generation $n$ as $t_{n}$, the phase in the forcing rhythm is $t_{n} / T_{\text {osc }}$, where $T_{\text {osc }}$ is the period of the forcing oscillator (approximately $24 \mathrm{~h}$ for the circadian clock). The model for analysis is

$$
\begin{gathered}
t_{n+1}^{ \pm}=t_{n}+\tau_{0}(1-\alpha)+\alpha T_{n-1}+k \sin \left(\frac{2 \pi t_{n}}{T_{\text {osc }}}\right)+\xi_{n}^{ \pm}, \\
T_{n}^{ \pm}=t_{n+1}^{ \pm}-t_{n},
\end{gathered}
$$

where $t_{n}$ represents the birth time of a cell in generation $n$, $T_{n}$ represents the cell-cycle duration of a cell in generation 
$n, \tau_{0}$ is the intrinsic cell-cycle period in the absence of circadian forcing, $k$ is a parameter that controls the magnitude of the coupling between the cell cycle and the circadian oscillation, $\alpha \in[-1,1]$ is a parameter that allows a tuning for the influence of the mother cell-cycle duration on the current cell-cycle duration. Instead of the cat map term introduced in Ref. [11], we add here a white noise term that is simpler and biologically more plausible, $\xi_{n}^{ \pm}$, with $\left\langle\xi_{n}^{ \pm}\right\rangle=0$ and $\left\langle\xi_{n}^{ \pm} \xi_{m}^{ \pm}\right\rangle=\xi^{2} \delta_{n, m} \delta_{+,-}$, where $\langle\cdot\rangle$ denotes average over realizations. The superscripts $( \pm)$ designate the two sister cells. If $\xi=0$, both sisters have the same cell-cycle duration. In this case, the model has a rich history. For $\alpha=0$, it is equivalent to the Arnold circle map [17]; for $\alpha=1$, it is the standard map [30]; and, for general values of $\alpha$, it is the fattened Arnold circle map [31], also known as the dissipative kicked rotor [32,33]. The latter has previously been considered only as an abstract model. Its properties, especially for $\alpha<0$, are not well understood. We find that, depending on the parameters, the dynamics of the model include fixed points (for example, around $\left.\tau_{0}=T_{\mathrm{osc}}\right)$ and regions of periodic, quasiperiodic, or chaotic behavior.

\section{RESULTS}

\section{A. Experimental correlations in lineages of cells}

We measured or analyzed the cell-cycle duration in thousands of single cells and in various organisms: E. coli, corynebacteria, cyanobacteria, EMT6 human cells, and L1210 mouse lymphocytes (Table I). While the first two organisms are not known to be controlled by an external oscillator, the circadian clock coupling to the cyanobacteria cell cycle has been extensively studied, and a similar coupling has more recently been suggested to be active in mammalian cells [26]. Corynebacteria were chosen because of their division mode that occurs by snapping (see Supplemental Material [8] for Video 1), thus reducing the experimental noise in the determination of the cell division event.

Mother cells divide into two daughter cells, called sisters. The sisters again divide into four cells. Two daughter cells from different sisters are called cousins (Fig. 1). As shown in our previous work on the analysis of the L1210 data [11], meaningful information can be gained from measuring the correlations between different cells within the same lineage. In particular, we measured the Spearman correlations in cell-cycle duration between sister cells $\rho_{s-s}$, between mother and daughter cells $\rho_{m-d}$, and between cousin cells $\rho_{c-c}$. (See Table I for measured correlations.) The averages in the correlation coefficients are over different lineages. We found significant correlations between sister cells in all data sets, in agreement with earlier results [36]. In most data sets, the $\rho_{m-d}$ was low or nonsignificant, suggesting that the memory of the cell-cycle duration was lost within one cell cycle. The coefficient of variation of the cell-cycle duration varied from $10 \%$ to $40 \%$ in the various data sets, similarly to typical values in the literature. This variation is larger, for example, than the one expected from the measured noise between sisters $\xi$, according to a simple model of inheritance, the bifurcation autoregression (BAR) model [37]. Another departure from this model is the observation that cousins often had correlated cell-cycle duration that could not be attributable to microenvironmental conditions [11]. Intuitively, the correlation between cousin cells despite the absence of correlation between mother and daughters is surprising. In order to formulate this intuition more rigorously and for the general case, we consider a process that proceeds in a treelike fashion, as the division process does [Fig. 1(a)].

\section{B. Expected correlation of cell-cycle duration in a lineage}

Similarly to the assumptions of the BAR model, we assume that the fate of a daughter cell is determined by inherited factors from its mother cell and that there are no external influences. Likewise, we assume that the fates of two sister cells are directly related due to the resemblance in their composition at birth. Thus, the correlations between cells are determined by the lineage relations.

In order to neutralize the effect of the fate of cell $\mathrm{Z}$ in Fig. 1(a), on the correlation of cells $\mathrm{X}$ and $\mathrm{Y}$, we use the

TABLE I. Typical experimental measures of the cell-cycle duration and correlations in various organisms (see also Table III). Mean cell-cycle duration computed on the third generation ( \pm std), coefficient of variation $(\mathrm{CV})$, Spearman correlations, and $\Delta$ [as defined by Eq. (7)]. The expected value for $\Delta$ for the null model is zero. The noise between sisters cells $\xi$ is defined in Eq. (1) and extracted from the data shown in Fig. 8 (see Sec. V). Data sets that have $\Delta$ significantly above zero are marked in grey (see Sec. V for significance evaluation).

\begin{tabular}{|c|c|c|c|c|c|c|c|c|c|}
\hline \hline Organism & Mean $(\mathrm{h})$ & $\mathrm{CV}$ & $\rho_{s-s}$ & $\rho_{m-d}$ & $\rho_{c-c}$ & $\Delta$ & Lineages & $\xi(\mathrm{h})$ & ref. \\
\hline Lymphocytes & $15.8 \pm 2.4$ & 0.15 & $0.88 \pm 0.02$ & $0.03 \pm 0.07$ & $0.60 \pm 0.05$ & $0.60 \pm 0.05$ & 87 & 0.75 & {$[10]$} \\
\hline EMT6 & $10.8 \pm 1.1$ & 0.10 & $0.8 \pm 0.05$ & $0.43 \pm 0.09$ & $0.77 \pm 0.05$ & $0.63 \pm 0.1$ & 41 & 0.51 & {$[35]$} \\
\hline Cyanobacteria 1 & $17.1 \pm 6.8$ & 0.40 & $0.91 \pm 0.04$ & $-0.33 \pm 0.10$ & $0.78 \pm 0.05$ & $0.68 \pm 0.1$ & 29 & 1.68 & {$[22]$} \\
\hline Corynebacteria & $1.56 \pm 0.38$ & 0.24 & $0.19 \pm 0.09$ & $-0.21 \pm 0.12$ & $-0.05 \pm 0.11$ & $-0.06 \pm 0.13$ & 51 & 0.36 & this work \\
\hline E.coli & $0.51 \pm 0.16$ & 0.31 & $0.35 \pm 0.09$ & $-0.06 \pm 0.25$ & $0.12 \pm 0.09$ & $0.12 \pm 0.1$ & 60 & 0.13 & this work \\
\hline \hline Cyanobacteria 2 & $14.4 \pm 3.2$ & 0.22 & $0.63 \pm 0.06$ & $-0.16 \pm 0.08$ & $0.34 \pm 0.07$ & $0.33 \pm 0.06$ & 65 & 2.09 & this work, $[36]$ \\
\hline Cyanobacteria 2 Mutant & $14.4 \pm 1.7$ & 0.12 & $0.41 \pm 0.08$ & $-0.17 \pm 0.08$ & $0.21 \pm 0.09$ & $0.20 \pm 0.08$ & 74 & 1.29 & this work, $[36]$ \\
\hline \hline
\end{tabular}


partial correlation function. The partial correlation $\rho_{\mathrm{XY}, \mathrm{Z}}$ between random variables $\mathrm{X}$ and $\mathrm{Y}$, removing the effect of the variable $\mathrm{Z}$, is

$$
\rho_{\mathrm{XY}, \mathrm{Z}}=\frac{\rho_{\mathrm{XY}}-\rho_{\mathrm{XZ}} \cdot \rho_{\mathrm{YZ}}}{\sqrt{\left(1-\rho_{\mathrm{XZ}}^{2}\right)\left(1-\rho_{\mathrm{YZ}}^{2}\right)}} .
$$

We now return to the labels of Fig. 1(a) and recall that we expect zero correlation between the fates of $\mathrm{X}$ and $\mathrm{Y}$ when the effect of $\mathrm{Z}$ is removed. For Spearman correlations, this assumption is valid if the correlations reflect monotonous relations between cells. Thus, $\rho_{\mathrm{XY}, \mathrm{Z}}=0$, and Eq. (3) implies

$$
\rho_{\mathrm{XY}}=\rho_{\mathrm{XZ}} \cdot \rho_{\mathrm{YZ}}
$$

Likewise, we expect zero correlation between $\mathrm{K}$ and $\mathrm{Y}$ when the effect of $\mathrm{X}$ is removed, so $\rho_{\mathrm{KY}, \mathrm{X}}=0$. Equation (5) is obtained directly from Eq. (4) by changing labels $(X \rightarrow K, Z \rightarrow X)$, and by substitution of Eq. (4):

$$
\rho_{\mathrm{KY}}=\rho_{\mathrm{KX}} \cdot \rho_{\mathrm{YX}}=\rho_{\mathrm{KX}} \cdot \rho_{\mathrm{XZ}} \cdot \rho_{\mathrm{YZ}} \cdot
$$

Replacing the labels KY, KX, XZ, and YZ by the cells' relations [as in Fig. 1(a)] leads to

$$
\rho_{c-c}=\rho_{m-d}^{2} \times \rho_{s-s} .
$$

This derivation is valid for any cell fate measured on a lineage. In particular, Eq. (6) is the expected relation between the cell-cycle correlations $\rho_{c-c}, \rho_{s-s}$, and $\rho_{m-d}$ (using Spearman coefficients), under the assumption of at most a monotonic dependence of a cell cycle on its mother cell cycle and on its sister cell cycle (see also [37]).

\section{Comparing expected and measured correlations in cell-cycle duration}

According to the derivation of the null model, $\rho_{c-c}$ is expected to be always smaller than $\rho_{m-d}$ [Eq. (6)]. For the data sets of corynebacteria and of E. coli, Eq. (6) holds quite well. However, for the cyanobacteria, lymphocytes, and EMT6 data sets, we observe a large deviation from this expected behavior (Fig. 2 and Table I), as quantified by the parameter $\Delta$ :

$$
\Delta=\rho_{c-c}-\rho_{m-d}^{2} \times \rho_{s-s}
$$

Moreover, in several data sets, contrary to expectations, we observe empirically an even stronger departure from Eq. (6), which we termed the cousin-mother inequality:

$$
\rho_{c-c}>\left|\rho_{m-d}\right| .
$$
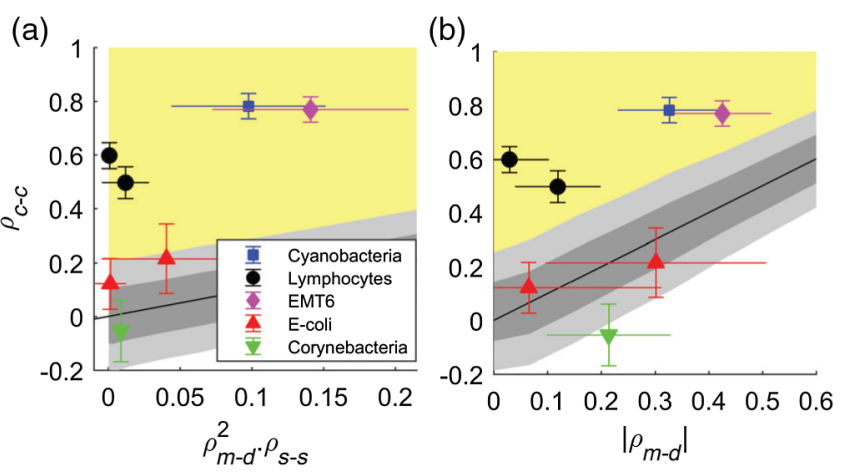

FIG. 2. Measured correlation coefficient in lineages. (a) Plot of the correlations in cell-cycle duration between cousin cells $\left(\rho_{c-c}\right)$ versus $\rho_{m-d}^{2} \times \rho_{s-s}$. The diagonal line corresponds to $\Delta=0$. Data points in the yellow area are those with $\Delta$ significantly above zero (p-value < 0.05), i.e., where Eq. (6) does not hold. (b) The same data as in (a), plotted as the correlations in cell-cycle duration between cousin cells versus $\left|\rho_{m-d}\right|$. Data points in the yellow area are those for which the cousin-mother inequality holds [Eq. (8)]. The grey areas mark correlation values that could result from a random process within one (dark grey) or two (light grey) standard deviations from the diagonal line (see Sec. V).

\section{Generic features of the kicked cell-cycle model}

The kicked cell-cycle model offers a potential explanation for these results, by including a nonmonotonous and nonlinear relation between the cell-cycle of the mother and its daughter, which departs from the assumptions of the null model. As the intrinsic cell-cycle period $\tau_{0}$, the forcing amplitude $k$, and the mother-daughter coupling $\alpha$ vary, different dynamical behaviors and bifurcations occur. In Fig. 3(a), the complex landscape of periodicity regions coming from the kicked cell-cycle model is shown for $\alpha=-0.5$. Despite the classical nature of this problem, there is still comparatively little known about the bifurcations when $\alpha \neq 0$ and $\alpha \neq 1$. We observed a strong similarity to the locking zones in the sine circle map for which $\alpha=0$ [21,22,38]. The rich nature of the dynamics shown in Fig. 4 is apparent also in the maximal Lyapunov exponent phase space of the kicked cell-cycle model that we plot in Fig. 5, which allows us to distinguish between chaotic (green to red), quasiperiodic (cyan), and periodic regions (blue). One can observe that these generic features are kept also when $\alpha$ is varied from negative [Fig. 5(a)] to positive [Fig. 5(b) and Fig. 6]. In order to find in which regions of the parameters' phase space the cousin-mother inequality observed in the data holds, we plot in Figs. 3(b) and 3(c) the simulated values of the cousin-mother inequality for noise level $\xi=0.01$ [Fig. 3(b)] and $\xi=0.1$ [Fig. 3(c)]. In a large part of the plot, the cousin-mother inequality holds, showing that the model can account for the departure of the experimental data from Eq. (6). This plot demonstrates that the Arnold tongue structure plays a strong role in defining the value of the correlations, even in the presence of a substantial noise level. Interestingly, the model 
(a)

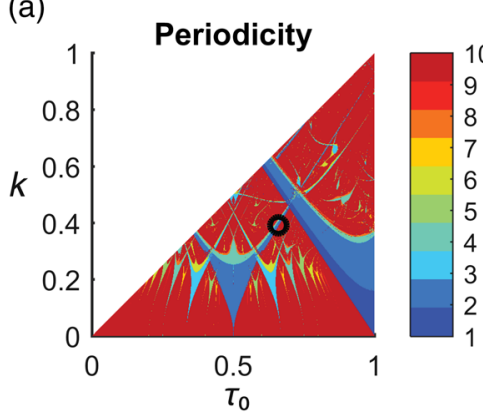

(b)



(c)

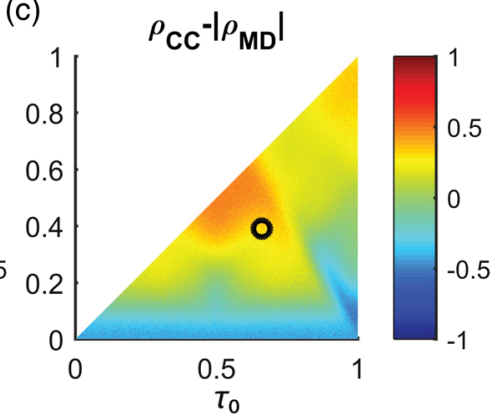

FIG. 3. Features of the kicked cell-cycle model with and without noise, $\alpha=-0.5$. (a) Periodicity map showing the Arnold tongues in the absence of noise. For example, dark blue denotes a fixed point region, light blue denotes a period 2 region, and dark red denotes either periods higher than 10 or quasiperiodic and chaotic regions. (b),(c) Visualization of the cousin-mother inequality [Eq. (8)] for a noise level of $\xi=0.01$ (b) and for $\xi=0.1$ (c), which represent the lower and upper bounds of the measured experimental noise (see Table II). The black circle denotes the fit parameters to the data set Cyanobacteria 1. $\tau_{0}, k$, and $\xi$ are in units of $T_{\text {osc }}$.

predicts that both negative and positive mother-daughter correlations can be obtained for the same coupling parameter $\alpha$ (Fig. 7), showing that $\alpha$ does not solely determine the sign of mother-daughter correlations.

\section{E. Fitting the mathematical model to the data}

In order to further test the validity of the kicked cellcycle model in describing the experimental data sets with high $\Delta$ (L1210, EMT6, and cyanobacteria), we extracted for each of these data sets the six measurements listed in Table I and fitted them to Eqs. (1)-(2) (see Sec. V for more details). The noise level of each data set was directly evaluated from the variation between sister pairs (see $\xi$ in Table I, Sec. V, for the derivation, and Fig. 8). The fit resulted in values for $\tau_{0}, k$, and $\alpha$ (Table II).

The values of $\tau_{0}$ are close to the measured mean values, but not identical, as expected from the model. The mean cellcycle duration is $\tau_{0}$ in the absence of a clock and is determined by growth conditions such as nutrients. However, the periodic forcing may drive the mean cellcycle duration to be above or below $\tau_{0}$, as seen in simulations [Fig. 9(a)]. Similarly, changing $T_{\text {osc }}$ can push the mean cellcycle duration below or above $\tau_{0}$ [see Fig. 9(b)].

Another interesting feature that comes out of the fitting procedure is that the cell-cycle distributions over the

TABLE II. Parameters' values [defined in Eq. (1)] extracted from the fitting procedure for the experiments with large $\Delta . \tau_{0}$ and $k$ are in units of $T_{\text {osc }}$ (we assumed $T_{\text {osc }}=24 \mathrm{~h}$ in all sets except Cyanobacteria 1; the measured value was $T_{\text {osc }}=23.16 \mathrm{~h}$ ). $\xi$ values are taken from Table I.

\begin{tabular}{lcccc}
\hline \hline Organism & $\tau_{0} \pm 0.01$ & $k \pm 0.01$ & $\alpha \pm 0.1$ & $\left(\xi / T_{\text {osc }}\right) \pm 0.01$ \\
\hline Lymphocytes 1 & 0.67 & 0.11 & 0.7 & 0.03 \\
Lymphocytes 2 & 0.65 & 0.18 & 0.7 & 0.06 \\
EMT6 & 0.45 & 0.06 & 0.9 & 0.02 \\
Cyanobacteria 1 & 0.66 & 0.39 & -0.5 & 0.07 \\
Cyanobacteria 2 & 0.59 & 0.17 & 0.2 & 0.09 \\
\hline \hline
\end{tabular}

population predicted from the simulations are very close to the measured distributions. One striking example is the cyanobacteria data (Cyanobacteria 1) that displays a bimodal distribution, and this bimodality is apparent also in the simulated distribution [Fig. 4(a)]. More examples of predicted distributions [Figs. 9(c) and 9(d)] show that changing $\tau_{0}$ or $k$ can result in switching between unimodal and bimodal distributions. Accordingly, as the parameters of growth of WT Cyanobacteria 2 are different from WT Cyanobacteria 1, the distribution of cell-cycle duration, which was bimodal for Cyanobacteria 1, is predicted by the model to be unimodal for Cyanobacteria 2, as observed [Fig. 9(e)].

The ability to determine the circadian phase at birth in single cells in Cyanobacteria enables us to compare the distribution of phases at birth to that obtained from simulations of the model. Interestingly, the distribution of the phase at birth of the cyanobacteria follows the predicted distribution from the model [Fig. 4(b)]. Note that the distribution of phases displays "gating"; i.e., the cells preferentially divide at certain phases of the clock [23]. This feature comes naturally out of the kicked cell-cycle model without having been fed into the model.

\section{F. Inheritance of cell-cycle duration in a mutant deleted for the clock genes}

The model suggests that the coupling of the cell cycle to the circadian clock can, depending on the parameters' values, either synchronize the cells and reduce cell-to-cell variability [39] or increase the variability of the cell-cycle duration in the population. In order to illustrate this prediction, we plot in Fig. 9(f) the simulated coefficient of variation $(\mathrm{CV})$ of the cell-cycle duration versus $k$. The $\mathrm{CV}$ increases with $k$ away from the locking regions. However, close to the locking regions, the $\mathrm{CV}$ decreases back. This suggests that driving the cell cycle by the circadian clock outside the fixed points' regions should lead to enhanced variability in the cell-cycle duration and, 
(a)



(b)

(c)

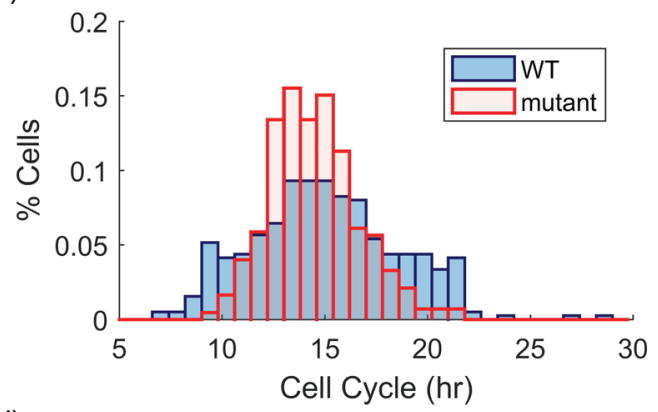

(d)

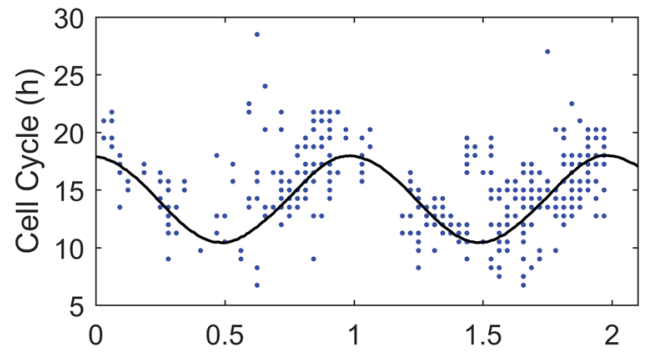

(e)

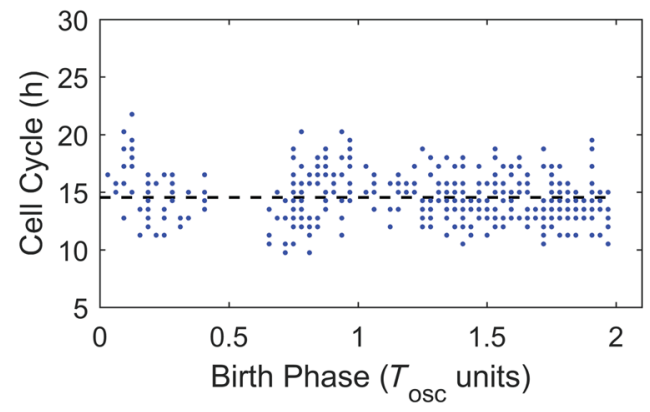

FIG. 4. Comparison between experiments and the kicked cellcycle predictions. (a) Distributions of the cell-cycle duration of Cyanobacteria 1, with data (blue bars) and model predictions (red lines). The simulated cell-cycle duration distribution is bimodal (red line), as the measured one. (b) Distribution of phase at birth for the same data as shown in (a). There is a reduction of cell division for certain phases, i.e., gating, as predicted by the model (red line). (c) Mutant of the strain of Cyanobacteria 2 deleted for the clock gene (red), displaying a significantly smaller coefficient of variation (CV) compared to the WT (blue). (d),(e) Cell-cycle duration as a function of time (in $T_{\text {osc }}$ units), synchronized to the circadian clock, for Cyanobacteria 2 (d) and a clock mutant (e), with data (blue dots) and model prediction [black line in (d)]. The dashed line in (e) represents the experimental mean of the mutant cell-cycle duration.

therefore, reducing the coupling to the circadian clock should reduce this variability. In order to test this prediction, we compared cyanobacteria cell-cycle variability for the wild-type (WT) strain and for a mutant deleted for the circadian clock. Here we present new data sets of WT strain of cyanobacteria and its derived clock mutant (Cyanobacteria 2 and Cyanobacteria 2 mutant in Tables I-II), as these two data sets were measured in the same conditions and have similar mean cell cycles (Table I). Interestingly, we observe that the variability in the cell cycle is significantly reduced (F-test, p-value $<0.05$ ) in the mutant strain $(\mathrm{CV}=0.12)$ compared to the WT strain $(\mathrm{CV}=0.22)$ [Fig. 4(c)]. Accordingly, whereas the cousin-mother inequality was fulfilled in the WT strain, it was not significant in the mutant strain (Table I).

A further direct comparison with the oscillating term predicted by the model can be done by plotting the experimental values of the cell-cycle duration versus the phase of the circadian clock at birth [Figs. 4(d) and 4(e)]. The oscillating dependence expected from the model [Fig. 4(d)] disappears in the mutant of the clock [Fig. 4(e)].

\section{DISCUSSION}

By fitting various data sets with the kicked cell-cycle model, we show that the model can reproduce the deterministic variability observed previously [11]. In addition, the model predicts several features such as nonmonotonic dependence of the mean cell-cycle duration on growth conditions (Fig. 9) and multimodal cell-cycle distributions (Fig. 9, [40]). Strikingly, although the kicked cell-cycle model does not explicitly gate the cell cycle at certain phases of the clock, effective gating occurs because of the dynamics of the coupling [Fig. 4(b)].

One main feature of the dynamics of the kicked cellcycle model is the observation that the cousin-mother inequality is obtained for quite a wide range of parameters, even when the noise level is relatively high [Fig. 3(c)]. However, there are still regions of parameters where the cousin-mother inequality is not fulfilled, for example, at fixed points. Also, higher noise level may eventually mask the deterministic periodic forcing, as well as the inequality, as illustrated in Fig. 8(f). Therefore, whereas the cousinmother inequality strongly suggests the existence of a nonlinear driving mechanism, its absence cannot lead to a definite conclusion. Although the absence of the inequality in E. coli and corynebacteria data is consistent with the absence of a known internal clock, a putative clock may have been masked by the factors mentioned above. Data of E. coli grown on a poorer medium $[3,41]$ shows the cousinmother inequality, and future work is needed to determine the underlying biological mechanism.

Comparison between the periodicity plot and the cousin-mother inequality plot reveals that both display the "Arnold tongues" features. The periodicity plot presented in Fig. 3(a) shows the geometry of the locking zones as the period $\tau_{0}$ and the amplitude of the periodic forcing $k$ are varied. This nonlinear locking can result in nonintuitive behavior of the driven system. For example, changing 
parameters can move the system from a period 1 (fixed point) to a period 2 zone, resulting in moving from a unimodal to a bimodal distribution of the cell-cycle duration, as shown in Fig. 9, and as observed [Fig. 4(a) and [40]].

Comparing the periodicity plot to the plot of the cousinmother inequality [Fig. 3(c)] reveals that, even in the presence of high noise level $(\xi=0.1)$, the period 2 and fixed point features are retained. At lower noise levels, it is interesting to follow which additional features are robust to noise. For example, the regions of intersections are very prominent in Fig. 3(b). Therefore, the strength of the cousin-mother inequality may serve as a straightforward experimental measurement that can reveal nontrivial features characteristic of discrete maps in empirical data.

In contrast with the periodicity plot, Lyapunov exponent, correlation dimension, or other indicators used to analyze chaotic behavior that require long data sets, the cousinmother inequality does not require extremely large quantities of data to detect deterministic contributions of the nonlinear process to the dynamics of the system. Therefore, the cousinmother inequality could be useful as a means to detect nonlinear coupling on lineages of cells. However, the analysis should be done only after experimental artifacts that may lead to similar observations are ruled out. In particular, spurious spatial correlation between cells due to the microenvironment should be ruled out by analysis of spatial and temporal dependencies. Furthermore, the departure from Eq. (6) may be due to noise and insignificant. Therefore, an analysis of the confidence interval for the departure from Eq. (6) should be done (see Sec. V), as represented by the grey areas in Fig. 2. Once these external influences are ruled out, the cousin-mother inequality can be a powerful tool for revealing the effect of nonlinear coupling on the cell-cycle variability. However, it should be noted that for determining a significant inequality (i.e., outside the grey area in Fig. 2), a large enough number of independent lineages should be analyzed. For example, another data set of cyanobacteria was analyzed [42], showing features consistent with a vicinity to the fixed point in the model (mean cell cycle close to $24 \mathrm{~h}$ ); however, the small number of lineages $(<20)$ resulted in nonsignificant correlations, and the fitting procedure could not be performed.

It should be noted that our analysis is not restricted to measurements of the cell-cycle duration. In effect, any observable that is measured on a self-replicating entity may display the cousin-mother inequality, provided that its variability is governed by a nonlinear process. For example, the cousin-mother inequality could be evaluated for correlations in the level of expression of specific genes or in the cell size increase, and it could detect nontrivial inheritance patterns. Therefore, we expect that the cousin-mother inequality could be used as a general indicator of deterministic nonlinear processes along lineages.

In this work, the cousin-mother inequality has been used to unveil a strong deterministic component in the variability of the cell-cycle duration of cyanobacteria. In agreement with our understanding, the deletion of the clock genes results in a significantly reduced cell-cycle variability in the population. In most theoretical analyses, such variability is understood as the inevitable consequence of cellular noise $[43,44]$. Here, we show that deterministic factors, such as periodic forcing, can increase the variability of the cell cycle in a way that can be controlled. In bacteria, the cellcycle variability has been shown to have important consequences for the survival of populations under stress [4], as well as for their ability to evolve resistance factors [45]. Therefore, understanding the source of this variability is important for predicting the behavior of bacteria under antibiotic treatment, as well as their ability to evolve.

\section{MATERIAL AND METHODS}

\section{A. Time-lapse microscopy}

\section{Lymphocytes}

The cells in Lymphocyte 1 and 2 were imaged in a Leica automated fluorescence microscope system, as previously described [11]. Briefly, a polydimethylsiloxane (PDMS) square mold was filled with medium (L-15) and sealed with a coverslip. Illumination was kept low enough to show no influence on total cell-cycle duration.

The analysis of division times was done using phasecontrast images and custom image analysis software (Fig. 1). We used an automatic cell-tracking platform written in MATLAB (MathWorks) as well as an ImageJ custom plug-in. Cell-cycle duration was determined from phase-contrast images acquired at 5-min intervals. Together with the sharp division process of L1210 cells, this resulted in less than $1 \%-2 \%$ experimental noise in $T_{n}$.

\section{E. coli}

In the E. coli experiments, we used the same system described for the lymphocytes. Here, the PDMS square mold was filled with melted Lysogeny broth (LB) agarose, that was prepared from LB Broth, Lennox (Difco) (LBL). Images were acquired using a $100 \times 1.3 \mathrm{NA}$ oil objective and a cooled CCD camera (Orca; Hamamatsu). Microscopy was carried out at $37^{\circ} \mathrm{C}$. The phase-contrast images were acquired at 1-min intervals. The images were tracked semiautomatically for supervised analysis using a plug-in developed for ImageJ [11].

Prior to microscopy, single colonies were diluted into fresh LBL. Cells were grown overnight at $37^{\circ} \mathrm{C}$, with shaking. Cultures were supplied with $15 \%$ glycerol and stored at $-80^{\circ} \mathrm{C}$. The frozen cultures were diluted into fresh $\mathrm{LBL}$ and grown at $37^{\circ} \mathrm{C}$, with shaking, for $3 \mathrm{~h}$.

\section{Cyanobacteria}

The Cyanobacteria 1 data set was described in Ref. [23]. Parameters used in this study for Cyanobacteria 2 were extracted from time-lapse movies of Synechococcus 
elongatus wild type cells (ATCC strain $33912^{\mathrm{TM}}$ ), and clock deletion mutant $(\triangle \mathrm{kaiBC})$ cells grown under constant light conditions [35]. Clock deletion cells carry an insertion of a gentamicin resistance cassette into the ORF of the kaiBC operon.

For time-lapse microscopy movies of cyanobacteria, cells were first grown in liquid BG-11 media at $30^{\circ} \mathrm{C}$ with constant rotation. The $\triangle \mathrm{kaiBC}$ strain was supplemented with gentamicin at $2 \mu \mathrm{g} \mathrm{mL}^{-1}$. Constant light levels were maintained at approximately $20-25 \mu \mathrm{E} \mathrm{m}^{-2} \mathrm{~s}^{-1}$ by cool fluorescent light sources. Cell cultures were kept at the exponential phase and entrained by subjecting cells to a 12-h light:12-h dark cycle. Therefore, the circadian phase was assumed to run freely after entrainment with a 24-h period, and the phase at birth was evaluated from the time elapsed since the release into constant light conditions. Nikon Ti-E inverted microscopes, equipped with the Nikon Perfect Focus System module, were then used to acquire time-lapse movies of growing microcolonies over several days under constant light at $15 \mu \mathrm{Em}^{-2} \mathrm{~s}^{-1}$, using a protocol adapted from Ref. [46]. Illumination for photoautotrophic growth was provided by a circular cool white light LED array (Cairn Research, U.K.), attached to the condenser lens. Images were acquired every 45 min using a CoolSNAP HQ2 camera (Photometrics, Arizona), and a $100 \times$ objective.

Movies were segmented and tracked using a modified version of Schnitzcells [47]. Finally, cell lineages were reconstructed by tracking individual cells across frames and identifying mother-daughter relationships in division events. For a full description of methods and data, see Ref. [35].

\section{Corynebacteria}

Experiments were carried out (as in Ref. [48]) using an inverted time-lapse live cell microscope (Nikon TI-Eclipse, Nikon Instruments, Germany) equipped with a $100 \times$ oil immersion objective (CFI Plan Apochromat Lambda DM 100×, NA 1.45; Nikon Instruments, Germany) and a temperature incubator (PeCon $\mathrm{GmbH}$, Germany). Cultivations were performed at $30^{\circ} \mathrm{C}$. Phase contrast images of growing microcolonies were captured every 2 min using an Andor Clara-E CCD camera (Andor Technology, U.K.).

Time-lapse movies were analyzed using a custom, specialized workflow implemented as an ImageJ/Fiji plug-in. Cell identification was performed using a segmentation procedure tailored to detect individual rod-shaped cells in crowded populations. Detected cells were subsequently tracked throughout all image sequences using an adapted single particle tracking approach as implemented in TrackMate. Derived quantities, i.e., growth rates, were computed using the Vizardous framework [49].

Cells were precultured as $20-\mathrm{mL}$ cultures in $100-\mathrm{mL}$ baffled Erlenmeyer flasks on a rotary shaker at 120-rpm orbital shaking at $30^{\circ} \mathrm{C}$. A first preculture in a brain-heart infusion (BHI) (Becton Dickinson, USA) complex medium was inoculated into a second preculture in a CGXII mineral medium, which was finally inoculated at OD600 $=0.05$ into a CGXII mineral medium, the main culture.

CGXII [50] was used as standard mineral medium for C. glutamicum cultivations consisting of (per liter) $20 \mathrm{~g}$ $\left(\mathrm{NH}_{4}\right)_{2} \mathrm{SO}_{4}, 5$ g urea, $1 \mathrm{~g} \mathrm{~K}_{2} \mathrm{HPO}_{4}, 1 \mathrm{~g} \mathrm{KH}_{2} \mathrm{PO}_{4}, 0.25 \mathrm{~g}$ $\mathrm{MgSO}_{4} \cdot 7 \mathrm{H}_{2} \mathrm{O}, 42 \mathrm{~g} 3$ morpholinopropanesulfonic acid (MOPS), $10 \mathrm{mg} \mathrm{CaCl}, 10 \mathrm{mg} \mathrm{FeSO}_{4} \cdot 7 \mathrm{H}_{2} \mathrm{O}, 10 \mathrm{mg}$ $\mathrm{MnSO}_{4} \cdot \mathrm{H}_{2} \mathrm{O}, 1 \mathrm{mg} \mathrm{ZnSO} \mathrm{Z}_{4} \cdot 7 \mathrm{H}_{2} \mathrm{O}, 0.2 \mathrm{mg} \mathrm{CuSO}{ }_{4}$, $0.02 \mathrm{mg} \mathrm{NiCl}{ }_{2} \cdot 6 \mathrm{H}_{2} \mathrm{O}, 0.2 \mathrm{mg}$ biotin, and $0.03 \mathrm{mg}$ of protocatechuic acid. The medium was adjusted to $\mathrm{pH} 7$ and $4 \%$ glucose $(\mathrm{w} / \mathrm{v})$ was added as a carbon source. All chemicals were purchased from Carl Roth and Sigma Aldrich. The medium was autoclaved and sterile filtered $(0.22-\mu \mathrm{m}$ pore size) to prevent clogging of the microfluidic channels by particles and cell agglomerates.

\section{B. Microfluidic devices: Corynebacteria}

Polydimethylsiloxane (PDMS) (Dow Corning; Farnell $\mathrm{GmbH}$, Germany) microfabrication was carried out [48] to manufacture single-use microfluidic devices with integrated $10-\mu \mathrm{m}$ high supply channels and cultivation chambers with a height of $1 \mu \mathrm{m}$. A $100-\mathrm{mm}$ silicon wafer (Si-Mat, Silicon Materials, Germany) was spin coated separately with two layers of SU-8 photoresist (Micro Resist Technology GmbH, Germany), processed by photolithography. This silicon wafer served as a reusable mold during subsequent PDMS casting. Thermally cured and separated PDMS chips were treated with oxygen plasma and permanently bonded to $170-\mu \mathrm{m}$-thick glass slides (Schott, Malaysia) just before the experiments. Manually punched inlets and outlets were connected with tubing (Tygon S-54-HL, ID = $0.25 \mathrm{~mm}, \mathrm{OD}=0.76 \mathrm{~mm}$; VWR International) via dispensing needles (dispensing tips, $\mathrm{ID}=0.2 \mathrm{~mm}, \mathrm{OD}=0.42 \mathrm{~mm}$; Nordson EFD Germany).

Fluid flow into the microfluidic chip was controlled with a four-fold NeMESYS syringe pump (Cetoni $\mathrm{GmbH}$, Germany). A detailed setup protocol can be found in Ref. [51]. Prior to microfluidic cultivation, the microfluidic chip was purged with fresh and sterile-filtered CGXII medium at $200 \mathrm{~nL} / \mathrm{min}$ for $10 \mathrm{~min}$. Afterwards, the chip was infused with a bacterial suspension for single-cell inoculation as described in full detail recently. Bacterial suspensions were withdrawn from the main culture at the exponential growth phase (OD600 between 0.5 and 1). As soon as sufficient single cells were inoculated into the microfluidic cultivation chambers, solely CGXII medium was infused at $200 \mathrm{~nL} / \mathrm{min}$ throughout the entire cultivation.

\section{Cell lines and bacterial strains}

\section{Lymphocytes}

L1210 lymphoblast cell line stably transfected with the Fucci marker system were used [11]. 


\section{E. coli}

E. coli B Rel606 strain was used.

\section{Cyanobacteria}

Cyanobacteria 1 was described in Ref. [23]. For Cyanobacteria 2 and the clock mutant, Synechococcus elongatus wild type cells (ATCC strain $33912^{\mathrm{TM}}$ ), and the corresponding clock deletion $(\triangle \mathrm{kaiBC})$ cells were used.

\section{Corynebacteria [48]}

Corynebacterium glutamicum ATCC 13032 strain was used.

\section{Computation of correlation coefficients}

All correlation coefficients are Spearman coefficients (similar results were obtained using Pearson coefficients). $\rho_{s-s}$ and $\rho_{c-c}$ were calculated on the third generation, while $\rho_{m-d}$ was calculated on the second and third generations (the mother from generation 2 and the daughter from generation 3). To avoid spurious dependencies, we were careful to include only one pair of cells chosen randomly from each cell lineage. The correlation coefficients were computed on the chosen cell cycles. This computation was repeated 1000 times, and the final correlation coefficients are the averages of those repetitions, while the errors of the correlations were taken to be the standard deviation $\sigma$.

\section{E. Significance computation}

We simulated cell cycles of random data. The simulated cells were taken from a normal distribution with experimentally measured coefficient of variation $(\mathrm{CV})$. Each simulation contained 60 lineages (the typical number of lineages that we track in each experimental set) and 7 cells per lineage (three generations, consisting of a mother, 2 sisters, and 4 cousins). By determining the covariance matrix of mothers and their two daughters, we matched $\rho_{s-s}$ and $\rho_{m-d}$ to the experimental results (with a separate simulation for each experiment). We computed all correlations from the simulated data and made 100 simulations for each experiment. We determined the departure from Eq. (6) as significant (with a p-value $<0.05$ ) if the experimental $\Delta$ (or the experimental value of $\rho_{c-c}-$ $\left.\left|\rho_{m-d}\right|\right)$ was larger than two standard deviations of the $\Delta$ (or $\rho_{c-c}-\left|\rho_{m-d}\right|$ ) that is obtained from the random simulations described above. The grey area in Fig. 2 was computed using 500 simulations, similar to the procedure described above. Here, we used constant typical values for $\mathrm{CV}$ and $\rho_{s-s}\left(\mathrm{CV}=0.15, \rho_{s-s}=0.6\right)$ and a range of values for $\rho_{m-d}$. The grey and light grey areas display one and two standard deviations from $\Delta=0$, respectively [Fig. 2(a)], or from $\rho_{c-c}=\left|\rho_{m-d}\right|$ [Fig. 2(b)]. These areas indicate the region where the correlations might result from a random process.

\section{F. Evaluation of $\boldsymbol{\xi}$ from the experimental data}

In Eq. (1), $\xi$ is the noise between sister cells that is related to the difference between sisters' cell cycle $\Delta T_{s s}$ (see Fig. 8):

$$
\Delta T_{s s} \equiv T_{n}^{+}-T_{n}^{-}=\xi_{n}^{+}-\xi_{n}^{-} .
$$

Thus, the variance of $\Delta T_{s s}$ is

$$
\operatorname{Var}\left(\Delta T_{s s}\right)=2 \xi^{2}
$$

We evaluate $\xi$ for each experiment, by computing the lefthand side of Eq. (10) from the data.

\section{G. Fitting method}

We simulated Eqs. (1) and (2) for a range of the parameters $\tau_{0}, k$, and $\alpha$, for 1000 lineages and 50 generations, and computed the correlations on the last generation. We fitted the model to six features of each experiment with $\Delta$ significantly above zero (see Table I, Fig. 2, and Sec. V 5): $\rho_{s-s}, \rho_{m-d}, \rho_{c-c}$, $\xi$, the mean cell cycle, and the cell-cycle $\mathrm{CV}$. We found all parameters that provide close features to the experimental features. We chose the parameters that provide the closest simulated $\rho_{s-s}$ as the best fit, as $\rho_{s-s}$ is the most significant measured correlation that we have, and we reckon that $\rho_{s-s}$ determines the noise level. For Cyanobacteria $1, T_{\text {osc }}$ was measured directly for every colony. We used the average on all colonies as the measured $T_{\text {osc }}$. For all other experiments, we fitted the model for $T_{\text {osc }}=24 \mathrm{~h}$.

\section{H. Periodicity computation}

In order to find the periodicity shown in Fig. 3(a), we simulated Eqs. (1) and (2), without noise, for 10,000 generations, and for a range of $\tau_{0}$ and $k$, with $\alpha=-0.5$. For the last 100 generations, we checked whether the circadian phase (which, in our case, is the absolute time modulo 1 , as we normalized all the parameters by $T_{\text {osc }}$ ) is equal to the previous generations. If the phase is equal to the phase of one generation before, the period is 1 . If not, but it is equal to the phase of two generations before, the period is 2, and so forth. Periods above 10 were not computed. The maximal Lyapunov exponent (Fig. 5) was computed as described in Ref. [52].

\section{ACKNOWLEDGMENTS}

We thank Qiong Yang and Alexander van Oudenaarden for retrieving data sets. This work was supported by grants from NSERC (L. G.), ERC Consolidator Grant No. 681619, Israel Science Foundation Grant No. 492/ 15 (N. Q. B.), Minerva Foundation, and the Lady Davis Visiting Professor fellowship (L. G.). A. G. (PD-311) and D. K. (VH-NG-1029) were supported by the German Helmholtz Association. 


\section{APPENDIX: ADDITIONAL FIGURES AND TABLE}

(a) Lyapunov Exponent

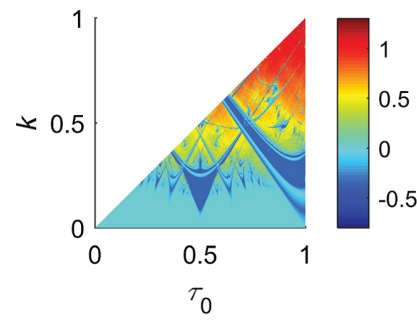

(b) Lyapunov Exponent

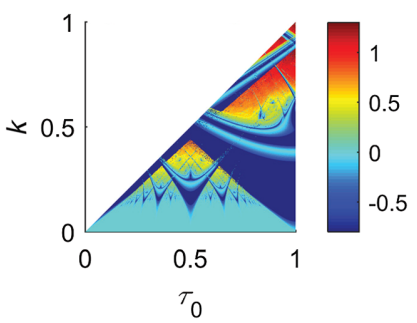

FIG. 5. Maximal Lyapunov exponent for $\alpha=-0.5$ (a) and $\alpha=0.2$ (b). The blue areas (negative Lyapunov exponent) are periodic areas. The cyan areas are quasiperiodic (zero Lyapunov exponent), and the red, yellow, and green represent the chaotic areas (positive Lyapunov exponent). The maximal Lyapunov exponent was computed as explained in Ref. [52]. The parameters in (a) are identical to those of Fig. 3(a), and similar features such as Arnold tongues are observed.

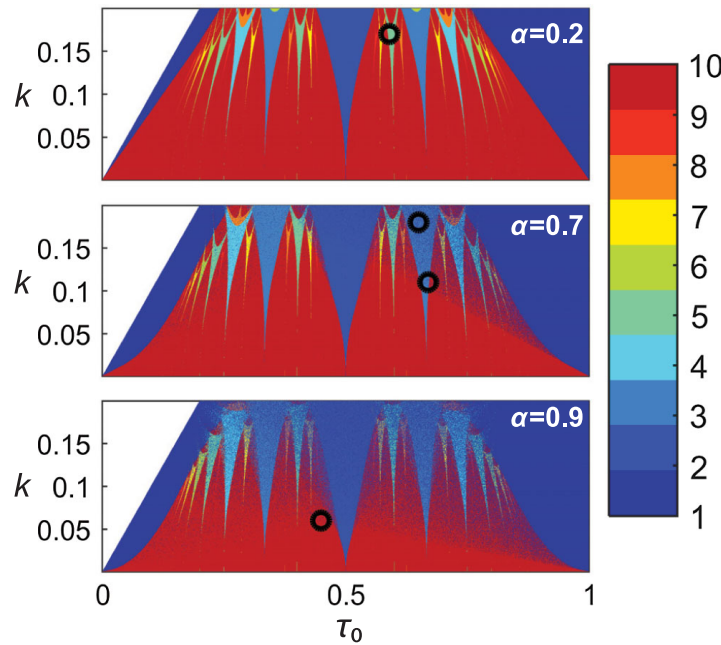

FIG. 6. Periodicity map showing the Arnold tongues in the absence of noise for the values of $\alpha$ extracted from the experimental data sets. For example, dark blue denotes a fixed point region, light blue denotes a period 2 region, and dark red denotes either periods higher than 10 or quasiperiodic and chaotic regions. The black circles indicate the values of $k$ and $\tau$ corresponding to the fit to the data (Cyanobacteria 2, lymphocytes, and EMT6 fitted parameters, with $\alpha=0.2,0.7$, and 0.9, respectively; see Table II). Note the similar features for the various $\alpha$, also similar for negative $\alpha$, as shown in Fig. 3(a).

TABLE III. Statistics of the cell divisions observed for the data shown in Table I.

\begin{tabular}{lcc}
\hline \hline Organism & Number of cells in third generation & Number of divisions \\
\hline Lymphocytes 1 & 321 & 580 \\
Lymphocytes 2 & 254 & 454 \\
EMT6 & 140 & 258 \\
Cyanobacteria 1 & 105 & 182 \\
Cyanobacteria 2 & 258 & 452 \\
Cyanobacteria mutant & 283 & 499 \\
Corynebacteria & 204 & 357 \\
E. coli 1 & 236 & 312 \\
E. coli 2 & 141 & 211 \\
\hline \hline
\end{tabular}




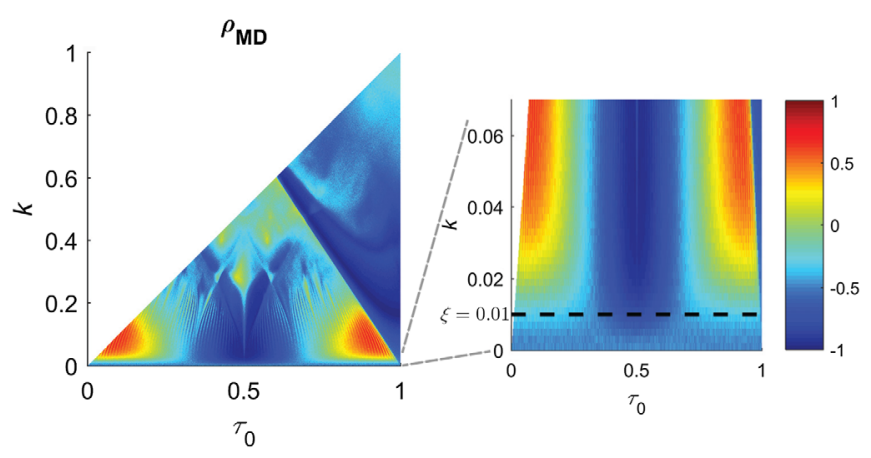

FIG. 7. Mother-daughter correlation $\rho_{\mathrm{MD}}$ as a function of $\tau_{0}$ and $k$, with noise level $\xi=0.01$ and $\alpha=-0.5$ [as in Fig. 3(b)]. Right: Zoom-in on small $k$ 's. It can be seen that for $k$ larger than the noise, $\rho_{\mathrm{MD}}$ can become positive, even for negative $\alpha$. For $k$ below the noise level (dashed black line), the forcing features disappear.
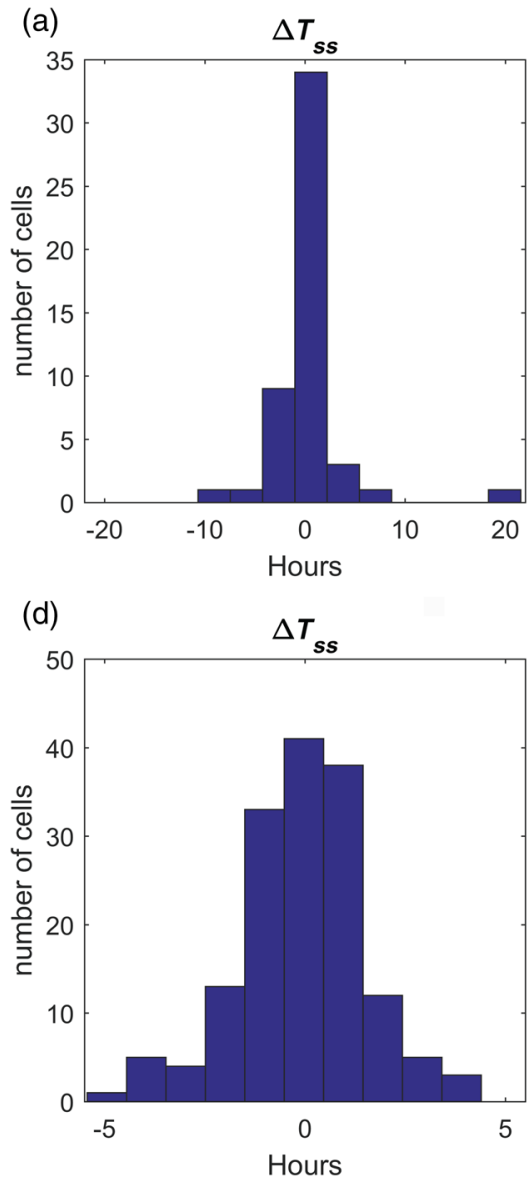

(b)

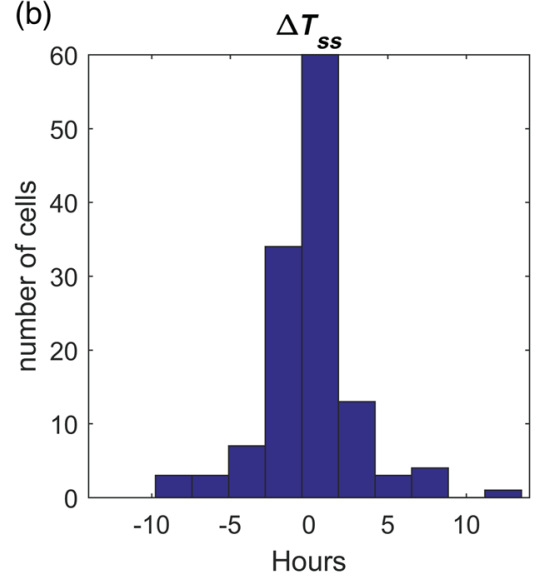

(e)

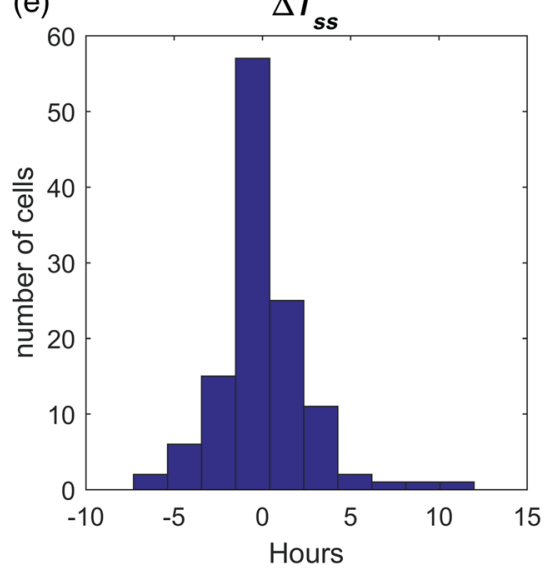

(c)

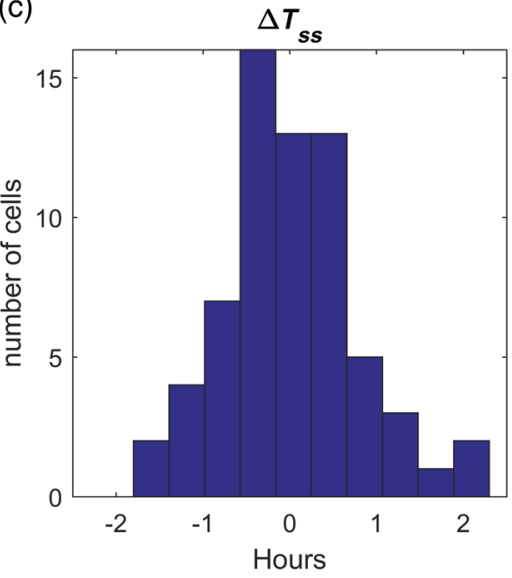

(f)

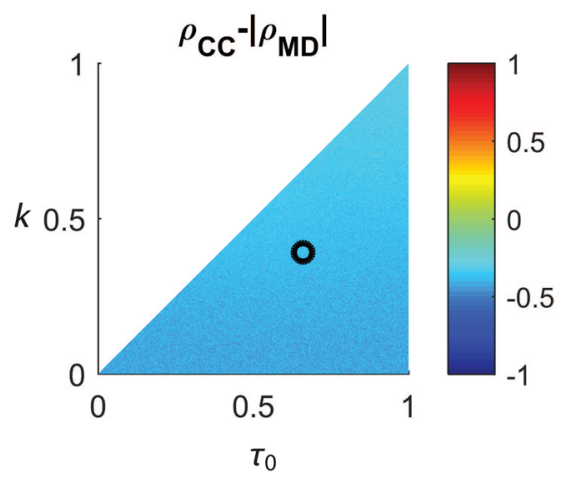

FIG. 8. Histograms of the experimental noise $\left[\Delta T_{s s}\right.$ from Eq. (9)] for all data sets with large $\Delta$ (Tables 1 and 2): (a) Cyanobacteria 1, (b) Cyanobacteria 2, (c) EMT6, (d) Lymphocytes 1, and (e) Lymphocytes 2. (f) Visualization of the cousin-mother inequality [Eq. (8)] for a noise level of $\xi=0.5$ and $\alpha=-0.5$. The parameters are the same as for Figs. 3(b) and 3(c), except for a greater noise level. Note that, at this high noise level, the periodic forcing features disappear and the cousin-mother inequality is not fulfilled. 
(a)

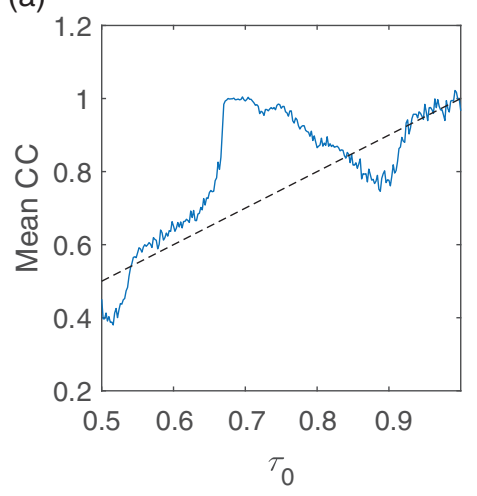

(c)

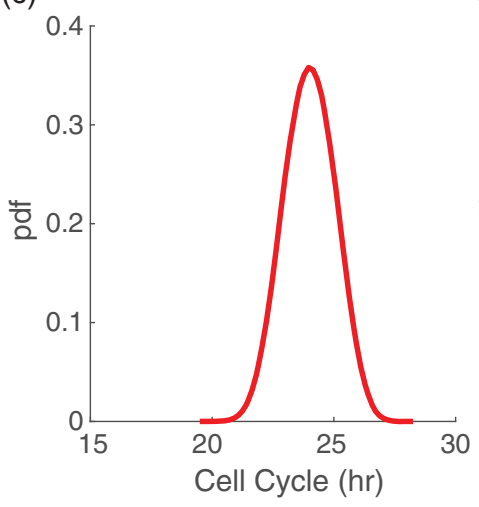

(f)

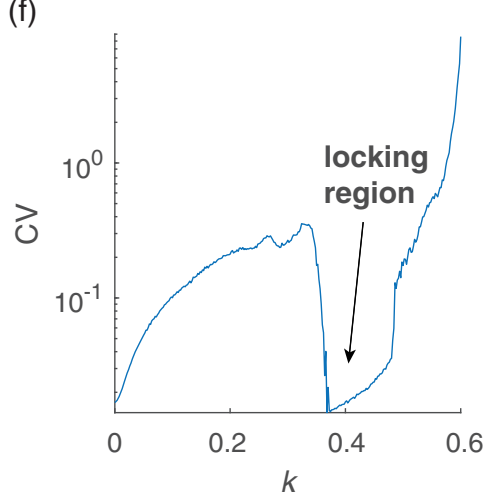

(b)

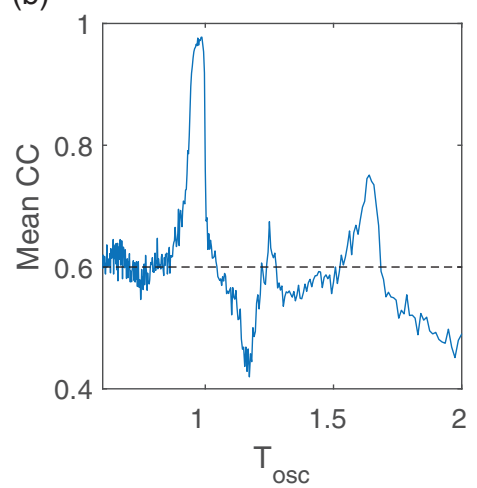

(d)

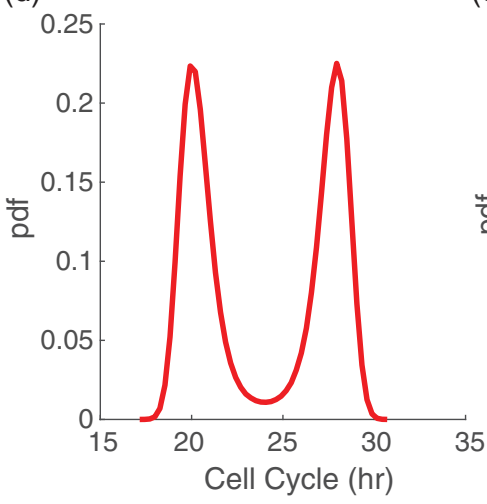

(e)

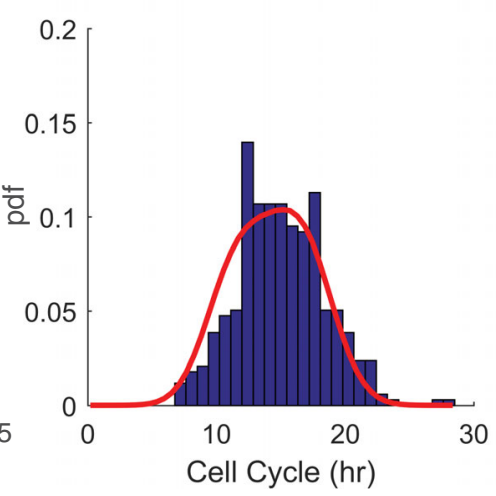

(g)

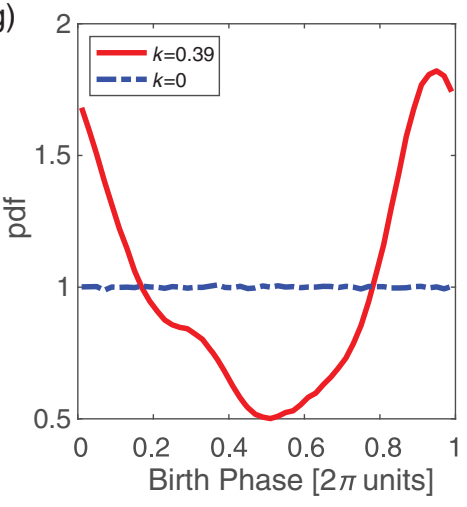

FIG. 9. Prediction of the kicked cell-cycle model. (a),(b) Nonmonotonous dependence of the mean cell-cycle duration versus different parameters. In both panels, $\alpha=-0.5$ and $\xi=0.01$. (a) $T_{\text {osc }}=1$ (accordingly, all times are in $T_{\text {osc }}$ units); $k=0.5$; and $\tau_{0}$ is varied. The dashed line is the line where the mean cell-cycle equals $\tau_{0}$. Paradoxically, changing $\tau_{0}$ from 0.7 to 0.9 (for example, by lowering the nutrients' concentration) can result in faster mean growth. (b) $k=0.6, \tau_{0}=0.6$ (the dashed line represents the value of $\tau_{0}$, and $T_{\text {osc }}$ is changing). (c),(d) Examples for cell-cycle duration distributions predicted by the model for different parameters. In both panels, $T_{\text {osc }}=24 \mathrm{~h}, k=0.4, \alpha=0.2, \xi=0.01 . \xi, k$, and $\tau_{0}$ are in $T_{\text {osc }}$ units. $\tau_{0}=0.8$ (c) or $\tau_{0}=1$ (d). (e) Distributions of the cell-cycle duration of experiment Cyanobacteria 2, with data (blue bars) and model predictions (red lines). (f) Cell-cycle duration CV versus $k$ as predicted from the model, with $\alpha=0.2, \tau_{0}=0.6$, and $\xi=0.01$. Note that the $\mathrm{CV}$ (and hence the variability) is increasing or decreasing for different $k$ 's. (g) Birth phase distribution as predicted from the model for the parameters obtained from the fit to Cyanobacteria 1 (Table 2) in the red line, and for the same parameters but with $k=0$ (blue dashed line). 
[1] P. R. Painter and A. G. Marr, Mathematics of Microbial Populations, Annu. Rev. Microbiol. 22, 519 (1968).

[2] A. Amir, Cell Size Regulation in Bacteria, Phys. Rev. Lett. 112, 208102 (2014).

[3] E. O. Powell, Some Features of the Generation Times of Individual Bacteria, Biometrika 42, 16 (1955).

[4] N. Q. Balaban, J. Merrin, R. Chait, L. Kowalik, and S. Leibler, Bacterial Persistence as a Phenotypic Switch, Science 305, 1622 (2004).

[5] P. Wang, L. Robert, J. Pelletier, W. L. Dang, F. Taddei, A. Wright, and S. Jun, Robust Growth of Escherichia Coli, Curr. Biol. 20, 1099 (2010).

[6] S. Hormoz, Z. S. Singer, J. M. Linton, Y. E. Antebi, B. I. Shraiman, and M. B. Elowitz, Inferring Cell-State Transition Dynamics from Lineage Trees and Endpoint SingleCell Measurements, Cell Syst. 3, 419 (2016).

[7] K. L. Frieda, J. M. Linton, S. Hormoz, J. Choi, K.-H. K. Chow, Z. S. Singer, M. W. Budde, M. B. Elowitz, and L. Cai, Synthetic Recording and In Situ Readout of Lineage Information in Single Cells, Nature (London) 541, 107 (2017).

[8] See Supplemental Material at http://link.aps.org/ supplemental/10.1103/PhysRevX.8.021035 for Video 1: time-lapse microscopy of Corynebacteria.

[9] A. A. Borbély, S. Daan, A. Wirz-Justice, and T. Deboer, The Two-Process Model of Sleep Regulation: A Reappraisal, Journal of sleep research 25, 131 (2016).

[10] M. R. Guevara, L. Glass, and A. Shrier, Phase Locking, Period-Doubling Bifurcations, and Irregular Dynamics in Periodically Stimulated Cardiac Cells, Science 214, 1350 (1981).

[11] O. Sandler, S. P. Mizrahi, N. Weiss, O. Agam, I. Simon, and N. Q. Balaban, Lineage Correlations of Single Cell Division Time as a Probe of Cell-Cycle Dynamics, Nature (London) 519, 468 (2015).

[12] J. Grasman, A Deterministic Model of the Cell Cycle, Bull. Math. Biol. 52, 535 (1990).

[13] M. Mézard and A. Montanari, Reconstruction on Trees and Spin Glass Transition, J. Stat. Phys. 124, 1317 (2006).

[14] G. Lambert and E. Kussell, Quantifying Selective Pressures Driving Bacterial Evolution Using Lineage Analysis, Phys. Rev. X 5, 011016 (2015).

[15] A. Pikovsky, M. Rosenblum, and J. Kurths, Synchronization: A Universal Concept in Nonlinear Sciences, (Cambridge University Press, Cambridge, England, 2003), Vol. 12.

[16] S. H. Strogatz, Nonlinear Dynamics and Chaos: With Applications to Physics, Biology, Chemistry, and Engineering (Westview Press, Boulder, 1994).

[17] V. I. Arnold, Geometrical Methods in the Theory of Ordinary Differential Equations (Springer Verlag, Berlin, 1983), Vol. 250.

[18] S. Smale, Differentiable Dynamical Systems, Bull. Am. Math. Soc. 73, 747 (1967).

[19] B. Chirikov and D. Shepelyansky, Chirikov Standard Map, Scholarpedia 3, 3550 (2008).

[20] H. Willaime, V. Barbier, L. Kloul, S. Maine, and P. Tabeling, Arnold Tongues in a Microfluidic Drop Emitter, Phys. Rev. Lett. 96, 054501 (2006).

[21] L. Glass, M. R. Guevara, J. Belair, and A. Shrier, Global Bifurcations of a Periodically Forced Biological Oscillator, Phys. Rev. A 29, 1348 (1984).
[22] L. Glass, Synchronization and Rhythmic Processes in Physiology, Nature (London) 410, 277 (2001).

[23] Q. Yang, B. F. Pando, G. Dong, S. S. Golden, and A. van Oudenaarden, Circadian Gating of the Cell Cycle Revealed in Single Cyanobacterial Cells, Science 327, 1522 (2010).

[24] C. Gérard and A. Goldbeter, Entrainment of the Mammalian Cell Cycle by the Circadian Clock: Modeling Two Coupled Cellular Rhythms, PLoS Comput. Biol. 8, e1002516 (2012).

[25] G. Charvin, F. R. Cross, and E. D. Siggia, Forced Periodic Expression of G1 Cyclins Phase-Locks the Budding Yeast Cell Cycle, Proc. Natl. Acad. Sci. U.S.A. 106, 6632 (2009).

[26] C. H. Johnson, Circadian Clocks and Cell Division: What's the Pacemaker?, Cell Cycle 9, 3864 (2010).

[27] T. Mori, B. Binder, and C. H. Johnson, Circadian Gating of Cell Division in Cyanobacteria Growing with Average Doubling Times of Less than 24 Hours, Proc. Natl. Acad. Sci. U.S.A. 93, 10183 (1996).

[28] T. Kondo, T. Mori, N. V. Lebedeva, S. Aoki, M. Ishiura, and S. S. Golden, Circadian Rhythms in Rapidly Dividing Cyanobacteria, Science 275, 224 (1997).

[29] J. Bieler, R. Cannavo, K. Gustafson, C. Gobet, D. Gatfield, and F. Naef, Robust Synchronization of Coupled Circadian and Cell Cycle Oscillators in Single Mammalian Cells, Mol. Syst. Biol. 10, 739 (2014).

[30] B. V. Chirikov, A Universal Instability of Many-Dimensional Oscillator Systems, Phys. Rep. 52, 263 (1979).

[31] H. Broer, C. Simó, and J. C. Tatjer, Towards Global Models near Homoclinic Tangencies of Dissipative Diffeomorphisms, Nonlinearity 11, 667 (1998).

[32] G. Zaslavsky and Z. Map, Scholarpedia 2, 2662 (2007).

[33] G. M. Zaslavsky, The Simplest Case of a Strange Attractor, Phys. Lett. 69A, 145 (1978).

[34] R. G. Staudte, M. Guiguet, and M. Collyn d'Hooghe, Additive Models for Dependent Cell Populations, J. Theor. Biol. 109, 127 (1984).

[35] B. M. C. Martins, A. K. Tooke, P. Thomas, and J. C. W. Locke, Cell Size Control Driven by the Circadian Clock and Environment in Cyanobacteria, DOI:.

[36] G. Hejblum, D. Costagliola, A.-J. Valleron, and J.-Y. Mary, Cell Cycle Models and Mother-Daughter Correlation, J. Theor. Biol. 131, 255 (1988).

[37] R. Cowan and R. Staudte, The Bifurcating Autoregression Model in Cell Lineage Studies, Biometrics 42, 769 (1986).

[38] L. Glass and R. Perez, Fine Structure of Phase Locking, Phys. Rev. Lett. 48, 1772 (1982).

[39] S. P. Mizrahi, O. Sandler, L. Lande-Diner, N. Q. Balaban, and I. Simon, Distinguishing between Stochasticity and Determinism: Examples from Cell Cycle Duration Variability, BioEssays 38, 8 (2016).

[40] G. Dong, Q. Yang, Q. Wang, Y.-I. Kim, T. L. Wood, K. W. Osteryoung, A. van Oudenaarden, and S.S. Golden, Elevated ATPase Activity of KaiC Applies a Circadian Checkpoint on Cell Division in Synechococcus Elongatus, Cell 140, 529 (2010).

[41] E. O. Powell and F. P. Errington, Generation Times of Individual Bacteria: Some Corroborative Measurements, Microbiology 31, 315 (1963).

[42] I. Mihalcescu, W. Hsing, and S. Leibler, Resilient Circadian Oscillator Revealed in Individual Cyanobacteria, Nature (London) 430, 81 (2004). 
[43] M. B. Elowitz, A. J. Levine, E. D. Siggia, and P. S. Swain, Stochastic Gene Expression in a Single Cell, Science 297, 1183 (2002).

[44] Y. Himeoka and K. Kaneko, Theory for Transitions between Exponential and Stationary Phases: Universal Laws for Lag Time, Phys. Rev. X 7, 021049 (2017).

[45] I. Levin-Reisman, I. Ronin, O. Gefen, I. Braniss, N. Shoresh, and N. Q. Balaban, Antibiotic Tolerance Facilitates the Evolution of Resistance, Science 355, 826 (2017).

[46] B. M. C. Martins, A. K. Das, L. Antunes, and J. C. W. Locke, Frequency Doubling in the Cyanobacterial Circadian Clock, Mol. Syst. Biol. 12, 896 (2016).

[47] J. W. Young, J. C. W. Locke, A. Altinok, N. Rosenfeld, T. Bacarian, P. S. Swain, E. Mjolsness, and M. B. Elowitz, Measuring Single-Cell Gene Expression Dynamics in Bacteria Using Fluorescence Time-Lapse Microscopy, Nat. Protoc. 7, 80 (2012).

[48] A. Grünberger, C. Probst, S. Helfrich, A. Nanda, B. Stute, W. Wiechert, E. von Lieres, K. Nöh, J. Frunzke, and D. Kohlheyer, Spatiotemporal Microbial Single-Cell Analysis
Using a High-Throughput Microfluidics Cultivation Platform, Cytometry A 87, 1101 (2015).

[49] S. Helfrich, C. E. Azzouzi, C. Probst, J. Seiffarth, A. Grünberger, W. Wiechert, D. Kohlheyer, and K. Nöh, Vizardous: Interactive Analysis of Microbial Populations with Single Cell Resolution, Bioinformatics 31, 3875 (2015).

[50] S. Unthan, A. Grünberger, J. van Ooyen, J. Gätgens, J. Heinrich, N. Paczia, W. Wiechert, D. Kohlheyer, and S. Noack, Beyond Growth Rate 0.6: What Drives Corynebacterium Glutamicum to Higher Growth Rates in Defined Medium, Biotechnol. Bioeng. 111, 359 (2014).

[51] A. Gruenberger, C. Probst, A. Heyer, W. Wiechert, J. Frunzke, and D. Kohlheyer, Microfluidic Picoliter Bioreactor for Microbial Single-Cell Analysis: Fabrication, System Setup, and Operation, J. Visualized Exp. 82, 50560 (2013).

[52] P. Langfield, W. L. C. Facanha, B. Oldeman, and L. Glass, Bifurcations in a Periodically Stimulated Limit Cycle Oscillator with Finite Relaxation Times, SIAM J. Appl. Dyn. Syst. 16, 1045 (2017). 